\title{
Wavelet-based ECG data compression optimization with genetic algorithm
}

\author{
Tsung-Ching Wu ${ }^{1,2}$, King-Chu Hung ${ }^{1}$, Je-Hung Liu ${ }^{1}$, Tung-Kuan Liu ${ }^{1}$ \\ ${ }^{1}$ National Kaohsiung First University of Science and Technology, Kaohsiung, Chinese Taipei \\ ${ }^{2}$ Tung Fang Design Institute, Kaohsiung, Chinese Taipei \\ Email: wtc@mail.tf.edu.tw
}

Received 12 May 2013; revised 15 June 2013; accepted 28 June 2013

Copyright (C) 2013 Tsung-Ching Wu et al. This is an open access article distributed under the Creative Commons Attribution License, which permits unrestricted use, distribution, and reproduction in any medium, provided the original work is properly cited.

\begin{abstract}
With a direct impact on compression performance, optimal quantization scheme is crucial for transformbased ECG data compression. However, traditional optimization schemes derived with signal adaption are commonly inherent with signal dependency and unsuitable for real-time application. In this paper, the variety of arrhythmia ECG signal is utilized for optimizing the quantization scheme of wavelet-based ECG data compression based on a genetic algorithm (GA). The GA search can induce a stationary relationship among the quantization scales of multi-resolution levels. The stationary property facilitates the control of multi-level quantization scales with a single variable. For this aim, a three-dimensional (3-D) curve fitting technique is applied for deriving a quantization scheme with linear distortion characteristic. The linear distortion property can be almost independent of ECG signals and provide fast error control. The compression performance and convergence speed of reconstruction quality maintenance are also evaluated by using the MIT-BIH arrhythmia database.
\end{abstract}

Keywords: Electrocardiogram; Error Control; Quantization Scale

\section{INTRODUCTION}

Electrocardiogram (ECG) is a non-invasive modality that senses electric action of heart motion from body surface. Being a three-dimensional (3-D) organ, heart disease diagnosis usually needs the use of multiple ECG signals sensed from different positions around the heart. Typical requirement is a record of 12-lead ECG signals [1]. ECG data compression technique is crucial for the transmission and long-term storage of mass ECG signals, e.g., ambulatory monitoring and telemedicine $[2,3]$. This technique can also benefit portable ECG recording due to low power consumption.

According to processed data, ECG data compression methods can be generally categorized into time-, transform-domain, and signal adaptation groups [4-6]. Signal adaptation methods based on a pre-process are unsuitable for real-time applications and time-domain methods are usually sensitive to the interference of high frequency components. With high compression performance and real-time processing capability, transform-domain methods have attracted much attention of researchers recently, especially for discrete cosine transform (DCT) [7] and discrete wavelet transform (DWT) [8] due to their excellent compression performance.

Transform-domain methods achieve data compression in terms of a non-uniform quantization scheme that uses different quantization scales for different frequency components. Quantization scheme will strongly impact on compression performance due to irreversible processing. For this sake, optimizing the compromise between compression ratio (CR) and distortion is crucial. Optimization schemes generally can be partitioned into filter selection and quantization scale design groups. With lattice parameterization, Nielsen et al. [9] proposed a signalbased optimization process that found optimal mother wavelet with minimal distortion rates for some fixed CRs. $\mathrm{He}$ and Mitra [10] designed optimal quantization error feedback filter by minimizing synthesis filtering error. Filter selection was also concerned for 3D signal compression [11]. For quantizing DCT coefficients, Batista et $a l$. [12] determined threshold and quantization vectors by minimizing the cost function $\mathrm{J}$ defined as a linear combination of entropy and distortion measure. BlancoVelasco et al. [13] built a nearly perfect reconstruction cosine modulated filter bank and determined threshold value with reconstruction quality guaranteed.

However, optimization schemes commonly need a 
pre-process for signal adaptation. This requirement is disadvantageous for real-time application. A less attractive approach was the set partitioning in hierarchical trees (SPIHT) algorithm [14] that combining quantization and coding can provide wavelet-based ECG data compressing with selectable bit rate. SPIHT scheme also has good compression performance. Considering the maintenance of reconstruction quality, an approach combining non-recursive discrete periodized wavelet transform (NRDPWT) and the reversible round-off linear transformation (RROLT) theorem was proposed [15]. The RRO-NRDPWT-based approach with the capability of error propagation resistance and octave coefficient normalization is efficient for reconstruction error control.

In this paper, a new quantization scheme that combines genetic algorithm (GA) and linear distortion program is presented for the RRO-NRDPWT-based ECG data compression. By using a test dataset involving 11 arrhythmia ECG signals, optimal quantization scales for a desired range is first found with a GA search. Using percentage root mean square difference (PRD) as the distortion measure, GA search with the criterion of minimum PRD/CR can induce a stationary relationship among multi-level quantization scales. This property implies that multi-level quantization scales can be controlled with single variable. Conducted by this hypothesis, a 3-D polynomial curve fitting technique is then applied for linear distortion program with respective to a generation variable $Q F$. Following the use of MIT-BIH arrhythmia database [16], linear distortion characteristic of the new quantization scheme, which benefits fast convergence in reconstruction quality maintenance, is evaluated. The experimental results of using untrained ECG signals also show that the GA-based quantization scheme can be independent of training data and improve average compression performance by $18.52 \%$ in comparing with the SPIHT scheme [14].

\section{QUANTIZATION SCHEME OF RRO-NRDPWT-BASED ECG DATA COMPRESSION}

RRO-NRDPWT is an efficient DWT process developed for easily controlling reconstruction error with minimum-word-length fixed-point computation. The fundamental architecture of RRO-NRDPWT-based ECG data compression system consists of three processes; i.e., RRO-NRDPWT, quantization, and lossless SPIHT encoding. The quantization scheme with single variable for controlling quantization scales of octave frequency bands can be independent of signals and suitable for real-time application. A brief review of the system is given in this section.

Let $\boldsymbol{S}_{\boldsymbol{J}}$ denote a row vector involving $N$-point sampled ECG data with $N=2^{-J}$ where $J<0$ is referred to as the decomposition level. For $J<j \leq 0$, the nonrecursive channel filter for the $j$ th level decomposition can be defined with a $N \times 2^{-j}$ matrix $\mathbf{A}_{j}$. In $\mathbf{A}_{j}$, the filter coefficients of two adjacent columns have a $2^{j-J}$. shift relationship in the vertical direction. By integrating the channel filters of all decomposition levels, a $N \times N$ filter-band matrix $\mathbf{A}=\left[\mathbf{B}_{0}, \mathbf{A}_{0}, \mathbf{A}_{-1}, \cdots, \mathbf{A}_{J+1}\right]$ can be constructed, where $\mathbf{B}_{0}$ is a column vector consisting of constant elements. Using matrix A, the 1-D RRO-NRDPWT can be represented with

$$
d^{*}=\left[s_{0}^{*}, d_{0}^{*}, d_{(-1)}^{*}, \cdots, d_{(J+1)}^{*}\right]=\left\lceil c_{\max }^{\prime} S_{J} A\right\rfloor,
$$

where $\lceil\mathbf{X}\rfloor$ denotes to round all the elements of vector $\mathbf{X}$ into the nearest integers, $c_{\max }^{\prime}$ is the significance normalization factor of $\mathbf{A}, \mathbf{d}_{j}^{*}$ is a row vector consisting of $2^{-j}$ integer wavelet coefficients of the $j$ th level, and $s_{0}{ }^{*}$ is a low-band coefficient of the terminate level. Matrix $\mathbf{A}$ with $\operatorname{det} \operatorname{det}(\mathbf{A})=1$ and $\mathbf{A}^{-1}=\mathbf{A}^{T}$ is a unitary matrix where $\mathbf{A}^{-1}$ and $\mathbf{A}^{T}$ denote the inverse and transport of $\mathbf{A}$, respectively.

The quantization scheme with generation variable $Q F$ can be defined by

$$
\overline{\mathbf{d}}_{j}^{*}=\left\lfloor\frac{\mathbf{d}_{j}^{*}}{c_{j}(Q F)}\right\rfloor \text { and } \overline{\mathrm{S}}_{0}^{*}=\left\lfloor\overline{\mathrm{S}}_{0}\right\rfloor=\left\lfloor\frac{\mathrm{S}_{0}^{*}}{c_{D C}(Q F)}\right\rfloor,
$$

where $\lfloor\mathbf{X}\rfloor$ denotes to truncate the elements of vector $\mathbf{X}$ into integers and $c_{j}(Q F)$ is the normalized quantization scale of the $j$ th level defined by

$$
\begin{aligned}
& c_{j}(Q F)=c p_{j}(Q F) / \operatorname{nap}_{j} \\
& \text { and } c_{D C}(Q F)=c p_{D C}(Q F) \operatorname{nap}_{D C}
\end{aligned}
$$

For inverse quantization, each retrieved datum will be compensated by half of the quantization scale, namely,

$$
\hat{\mathbf{d}}_{j}=c_{j}(Q F) \times\left(\overline{\mathbf{d}}_{j}^{*}+0.5 \operatorname{sign}\left(\overline{\mathbf{d}}_{j}^{*}\right)\right),
$$

where $\hat{\mathbf{d}}_{j}$ is the $j$ th sub-band vector of $\hat{\mathbf{d}}$ and $\operatorname{sign}(X)$ denotes the sign vector of $\mathbf{X}$, e.g., $\operatorname{sing}([-5,3])$ $=[-1,1]$. The quantized data $\overline{\mathrm{S}}_{0}^{*}$ and $\overline{\mathbf{d}}_{j}$ will be encoded with the differential pulse code modulation (DPCM) and lossless SPIHT scheme, respectively.

\section{OPTIMAL QUANTIZATION SCHEME DESIGN BASED ON GENETIC ALGORITHM}

GA invented by J. Holland [17] is a global searching method commonly used for finding optimal solutions of multi-variable non-linear system. This method achieves global searching based on uniformly distributed population $[18,19]$ of training data and finds solutions by three stratagems, i.e., selection, crossover, and mutation. Selection defines the criterion of candidate selection, crossover acts as a filter with clustering effect, and mutation 
is used to prevent from a trap of local sink. For overcoming data dependency of the GA-based optimization, it is desirable to use diversified training data. For this purpose, 11 arrhythmia ECG signals (i.e., record number $100,101,102,103,107,109,111,115,117,118$, and 119) saved in the MIT-BIH database are selected to build a training dataset. Each signal with 360 sampling rate and 11 bits resolution involves 10-min length sampled data. The quantization scheme design consists of two stages. The first stage applies GA to find four sets of optimal quantization scales where each set involves 11 level quantization scales, i.e.,

$\left\{c p_{D C}(), c p_{0}(), c p_{-1}(), \ldots, c p_{-8}(), c p_{-9}()\right\}$. Each set of multi-level quantization scales acts as a seed that will result in a PRD value for each signal during range $[0.5 \%$, $7 \%$ ]. The four seeds corresponding to four specified $c p_{-9}()$ (i.e., $c p_{-9}()=16,8,4$, and 2 ) will be found with minimum $\mathrm{PRD} / \mathrm{CR}$ being the selection criterion where $C R=$ (bits of compressed signal)/(bits of originalsignal). The compressed data file of each signal consists of a $Q F$ value and quantized ECG data. The former was encoded with the DPCM method and the latter was encoded with lossless SPIHT scheme. The GA-based searching process is described in the following:

\begin{tabular}{ll}
\hline Objective: & Find the values of $c p_{D C}(Q F)$ and $c p_{j}(),-8 \leq j \leq 0$, \\
for $c p_{-9}()=2,4,8$, and 16, respectively. \\
Fitness function: & Minimizing the ratio of PRD/CR. \\
Initialization: & Generate 100 data sets randomly with each set \\
defined as $\left\{c p_{D C}(), c p_{0}(), c p_{-1}(), \cdots, c p_{-8}()\right\}$. \\
Evolution Rules: \\
Selection: \\
Eighty sets with smallest PRD/CR are selected for \\
crossover in each iteration. \\
1) The choice of $\left\{c p_{D C}(), \cdots, c p_{j}()\right\}$ or $\left\{c p_{j-1}(), \cdots\right.$, \\
$\left.c p_{-8}()\right\}$ for crossover is random where the value of $j$ \\
is also randomly chosen and the choosing \\
probability is defined by 0.5. \\
2) Crossover processing number for each iterationis \\
defined as 40. \\
1) Mutation process is defined as exchanging the \\
Mutation: & values of $c p_{i}()$ and $c p_{j}()$ when they are selected. \\
2) The mutation probability is defined by 0.3. \\
1) Iteration times should exceed 100. \\
2) Select the set with maximum $c p_{-8}()$ and \\
terminate the iteration.
\end{tabular}

In the algorithm, a large mutation probability (i.e., 0.3) is selected for the desire of fast convergence. As shown in Figure 1, the $c p_{j}()$ solutions of ECG signal 101 have the trend of $c p_{i}()<c p_{j}()$ for $i>j$. In fact, this trend is also true for all ECG signals. This trend implies that for a specific $c p_{-9}()$, GA search can always end at a globally optimal solution that selects one set of multi-level quantization scales $\left(c p_{j}(), 0 \geq j \geq-8\right)$ with minimum $\mathrm{PRD} / \mathrm{CR}$. This result cannot be influenced by mutation probability. For the training dataset, $44 c p_{j}()$ values for each $j$ will be found. Figure 2

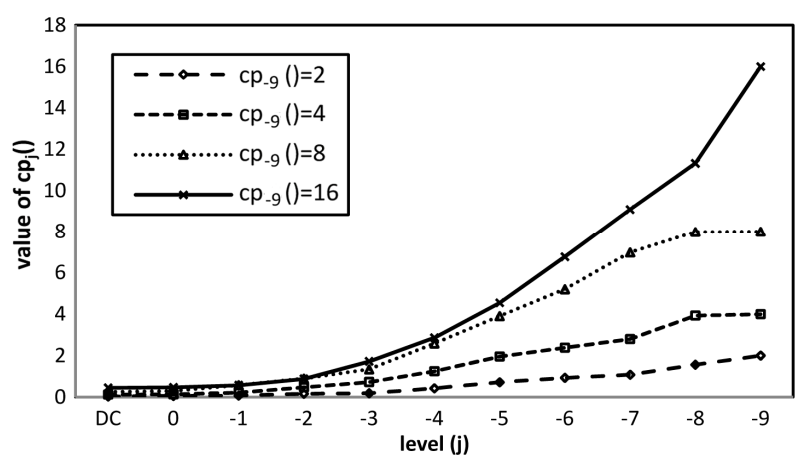

Figure 1. The GA-based $c p_{j}($ ) of the ECG signal 101 for four specified.

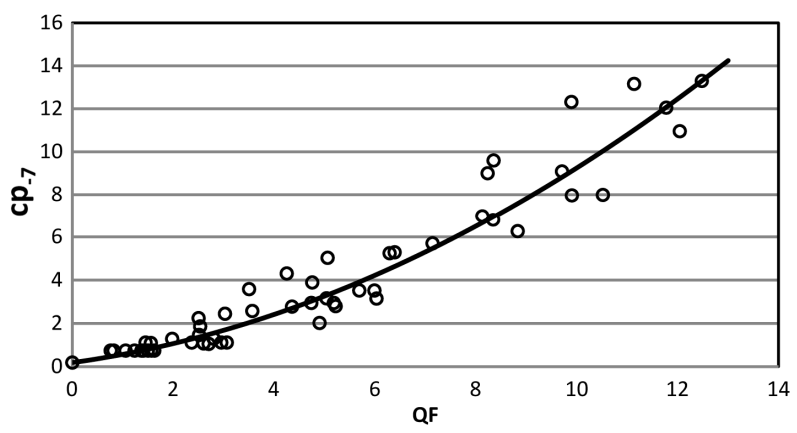

Figure 2. Curve fitting result of $c p_{-7}(Q F)$ by using the training dataset.

shows the 44 values of $c p_{-7}()$.

The GA solutions also imply that the criterion minimum $\mathrm{PRD} / \mathrm{CR}$ can induce a stationary relationship among multi-level quantization scales, i.e.,

$c p_{i}()=\alpha_{i j} c p_{j}()$ with constant $\alpha_{i j}$. This property can rationalize the control of all $c p_{j}()$ with a single variable. Based on the hypothesis, the second stage is to create a $c p_{j}()$ generation function by applying 3-D curve fitting technique. The 3-D coordinate system consists of three real number axes defined with PRD, CR, and $c p_{j}()$, respectively. For simplicity, the generation function is approximated with

$c p_{j}(Q F)=a_{j} Q F^{2}+b_{j} Q F+c_{j}$ where $Q F$ being the control variable is defined with $Q F=\sqrt{P R D^{2}+C R^{2}}$. For dealing with low $P R D$ region, the curve fitting also involves two new sets of low $C R$ case. One uses constant $c p_{j}()=n a p_{j} \times c_{\max }^{\prime}$, this case will introduce $11 C R$ values with $P R D=0$ for the 11 ECG training signals. The other uses $c p_{j}()=n a p_{j}$. Combining the two cases, the training dataset will provide 663 -D points for the curve fitting of each $c p_{j}(Q F)$. By using the least square error (LSE) method, the 10-level coefficients $a_{j}, b_{j}$, and $c_{j}$ for $j=0, \cdots,-9$ can be found as follows:

$$
\begin{aligned}
& c p_{D C}(Q F)=0.001974 \times Q F^{2}+0.01451 \times Q F+0.008015 \\
& c p_{0}(Q F)=0.001974 \times Q F^{2}+0.0198 \times Q F+0.01412
\end{aligned}
$$




$$
\begin{aligned}
& c p_{-1}(Q F)=0.002712 \times Q F^{2}+0.03241 \times Q F+0.01997 \\
& c p_{-4}(Q F)=0.01613 \times Q F^{2}+0.1544 \times Q F+0.07405 \\
& c p_{-5}(Q F)=0.02507 \times Q F^{2}+0.2209 \times Q F+0.1033 \\
& c p_{-6}(Q F)=0.04367 \times Q F^{2}+0.241 \times Q F+0.1422 \\
& c p_{-7}(Q F)=0.05839 \times Q F^{2}+0.322 \times Q F+0.1897 \\
& c p_{-8}(Q F)=0.0661 \times Q F^{2}+0.4167 \times Q F+0.2384 \\
& c p_{-9}(Q F)=0.0661 \times Q F^{2}+0.5122 \times Q F+0.2506
\end{aligned}
$$

Figure 2 illustrates the curve fitting result of $c p_{-7}(Q F)$. The quantization scales of $c p_{-j}(Q F)$ for $j=0 \sim-9$ and $Q F=1 \sim 15$ are depicted in Figure 3. This result shows that the proposed quantization scheme design method can effectively prevent from obtaining an exponential $c p_{j}(Q F)$ growth in high CR region.

\section{EXPERIMENTAL RESULTS}

In this section, compression performance and data dependency of the GA-based quantization scheme referred to as NRDPWT-GAar are studied. All experiments were performed on an IBM PC with Microsoft Windows 7, Intel Core $i 72.8 \mathrm{GHz} \mathrm{CPU}$ and $8 \mathrm{~GB}$ RAM. For compression performance comparison, 48 arrhythmia ECG signals in MIT arrhythmia database were used. Each signal involves 10-min length sampled data. Figure 4 shows the average PRD- $Q F$ and CR- $Q F$ curves of NRDPWT-GA, both are approximately linear. In comparing with the local optimal quantization scheme NRDPWT-6t, [2] the NRDPWT-GA has smoother $c p_{j}(Q F)$ curve in high $\mathrm{CR}$ region. On the other hand, the NRDPWT-6t has an exponential $c p_{j}(Q F)$ growth in high CR region. This improvement can result in NRDPWT-GA with more linear distortion behavior, especially for high CR case. By using 48 arrhythmia ECG signals, three wavelet based approaches are compared in Figure 5 where NRDPWT-GA with optimal quantization scale design can obtain the best compression performance in both low and high CR regions. In comparing with SPIHT scheme, the compression performance can be improved by $12.26(\%)$ and $10.13 \%$ for the two ranges 4 $\leq C R \leq 12$ and $14 \leq C R \leq 20$, respectively.

Generally, data dependency can be an intrinsic property of GA-based approach. This property can lead to instable result in practical applications. For studying the data dependency effect of GA-based quantization scheme, a second training dataset comprising 8 ECG signals randomly selected from MIT-BIH ST change database was build. These signals were the records 301, 305, 310, 314, $315,317,325$ and 326 with each involving 10-min length sampled data. By applying the same training process of GA and 3-D curve fitting described in Section 3, a second

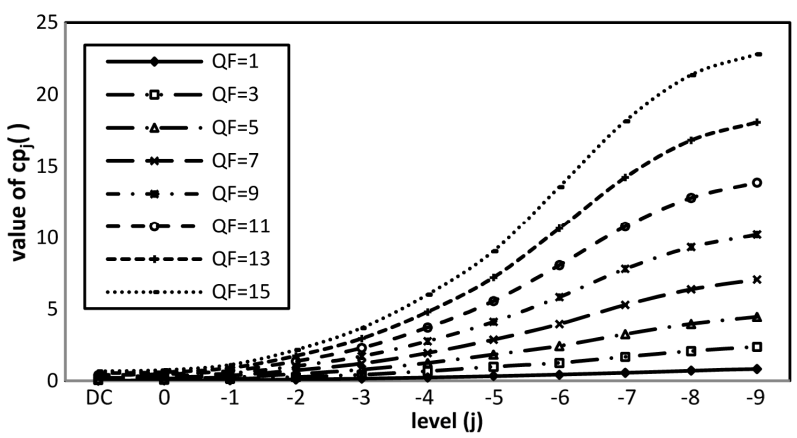

Figure 3. The $c p_{j}(Q F)$ values of the GA-based quantization scheme.

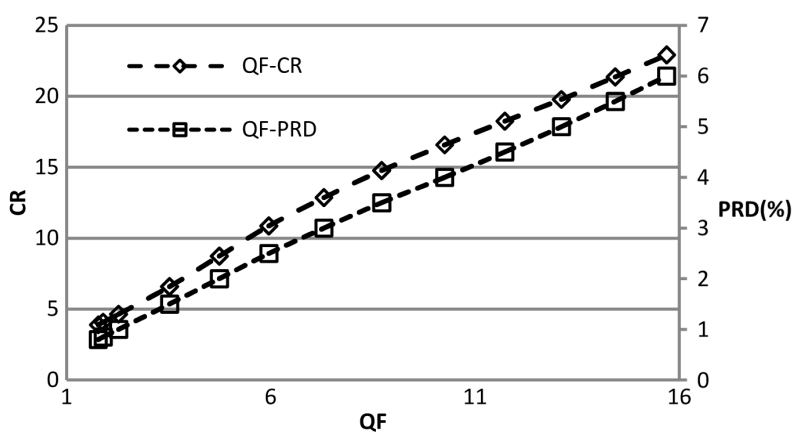

Figure 4. Compression performance of the GA-based quantization scheme for the 48 ECG signals in MIT arrhythmia database.

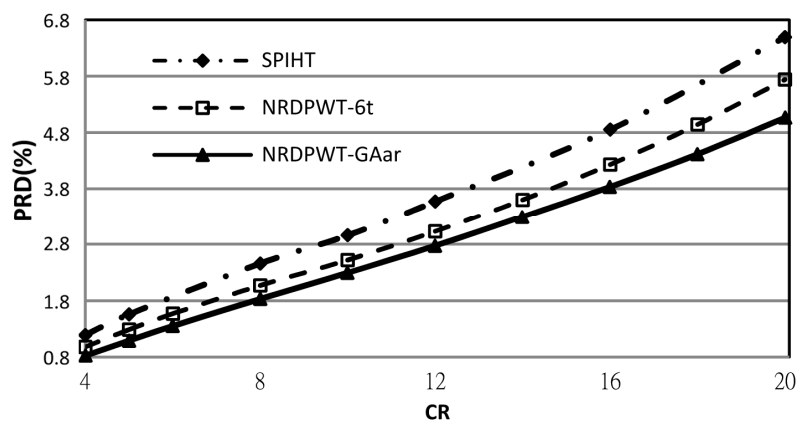

Figure 5. Compression performance comparison of three wavelet-based approaches.

GA-based quantization scheme referred to as NRDPWTGAst is derived in Figure 6 where the robustness of exponential-growth resistance in high $\mathrm{CR}$ region is also obvious. For a comparison of the two GA-based quantization schemes, all the signals saved in arrhythmia database and ST change database were used. The former and later comprise 48 and 28 ECG signals, respectively. Each signal involves 15-min length sampled data. The evaluation results were shown in Figures 7-9 where both the two schemes can obtain approximately linear distortion results with very minor difference. The comparison implies that data dependency effect of GA-based quantization scheme can be almost neglected in practice. Figure 8 shows that NRDPWT-GAar using arrhythmia signal is 


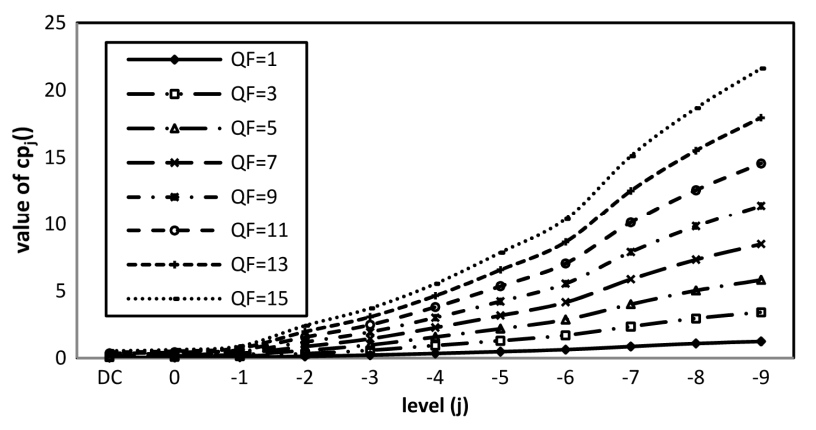

Figure 6. The $c p_{j}(Q F)$ values of NRDP-WT-GAst quantization scheme.

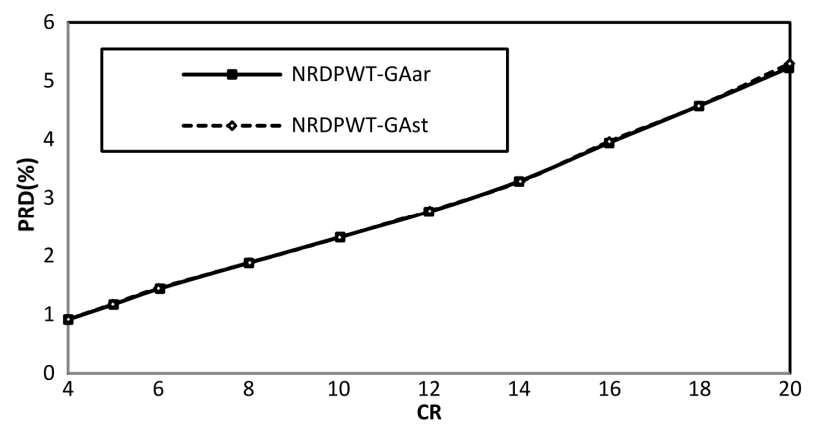

Figure 7. Compression performances of two GA-based quantization schemes by using MIT-BIH arrhythmia database.

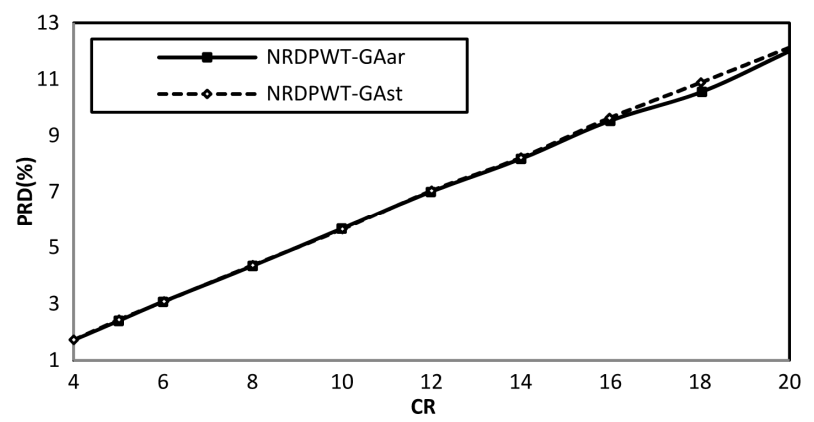

Figure 8. Compression performances of two GA-based quantization schemes by using MIT-BIH ST change database.

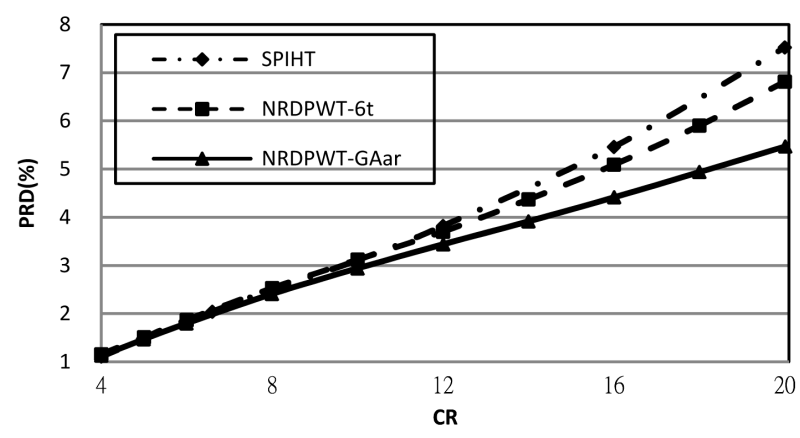

Figure 9. Compression performance comparison of three wavelet-based approaches with untrained noisy ECG signals.

slightly better than NRDPWT-GAst. An exploration using untrained noisy signals (i.e., records 104, 107, 111, 112 , 115, 116,117, 118, 119, 201, 207, 208, 209, 212, 213,
$214,228,231$, and 232) was also taken. Each signal contains about 1-min length sampled data. Three waveletbased approaches were compared in Figure 9 where NRDPWT-GAar also can obtain the best compression performance. In comparing with SPIHT scheme, the compression performance can be improved by $6.19(\%)$ and $27.85 \%$ for the two ranges $4 \leq \mathrm{CR} \leq 12$ and $14 \leq \mathrm{CR}$ $\leq 20$, respectively.

Quantization scheme with linear distortion characteristic can not only obtain high compression performance, but also facilitate in reconstruction quality maintenance that can be fulfilled with a close-loop error control process [20]. For exploring the quality maintenance performance of NRDPWT-GAar, the three ECG signals, records 109, 117, and 232, were used. From Figure 4, an approximately linear relationship between $P R D$ and $Q F$ can be derived by LSE method as

$P R D=0.417 Q F-0.07717 \cong 0.4138 Q F$. Applying this relationship into the linear $Q F$ prediction model used in the close-loop error control process can obtain the ECG data compression results shown in Figures 10-12, respectively. The three figures only demonstrated the first 316 segments of each file where $P R D_{T}$ denotes the target $P R D$ and $\varepsilon=\left|P R D-P R D_{\mathrm{T}}\right| / P R D_{\mathrm{T}} \times 100 \%$ is the fault tolerance of error control. Record 109 has a waveform with baseline wandering and slightly noise coupling. Figure 10 shows that a stable low bit rate $(\mathrm{CR} \approx 20)$ can be obtained. The desired $Q F$ can be also stable and convergence speed is very fast, even for a suddenly violent rate change. The iteration times (ITs) of error control process are distributed in the dynamic range $[1,3]$. The two segment demonstrations of Figures 10(e) and (f) show that the clinical information including the amplitude and duration can be preserved well. Record 117 is a nice waveform ECG signal. Figure 10 shows that for violent $Q F$ changes, the convergence speed can be still fast. The dynamic range of ITs is $[1,4]$. The reconstructed signal with very low rate $(\mathrm{CR} \approx 24)$ has slightly distortion at the boundary of segments. This can be overcome by reducing $P R D_{T}$. As shown in Figure 12, record 232 is a distorted and noisy waveform ECG signal. Though both rate and $Q F$ are violently changed, the dynamic range of ITs can be maintained in $[1,4]$. This signal has poor $P R D$ due to the smoothing effect of quantization process, but the reconstruction error is almost unobservable.

\section{CONCLUSION}

For wavelet-based ECG data compression, the problem of quantization scheme optimization was studied by using two kinds of ECG signals and a GA search in this paper. The experimental results showed that GA-based quantization scheme can be inherent with the property of signal independency. Using a training set involving more 


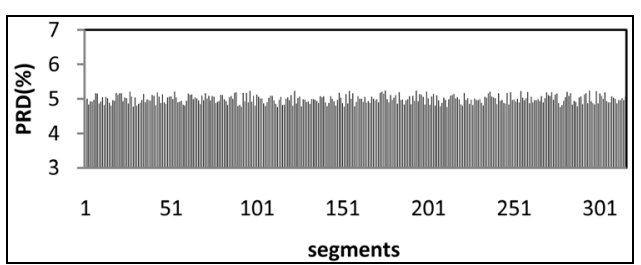

(a)

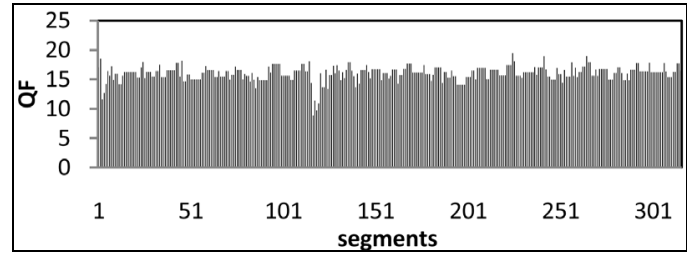

(c)

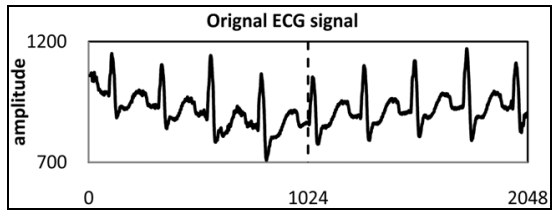

(e)

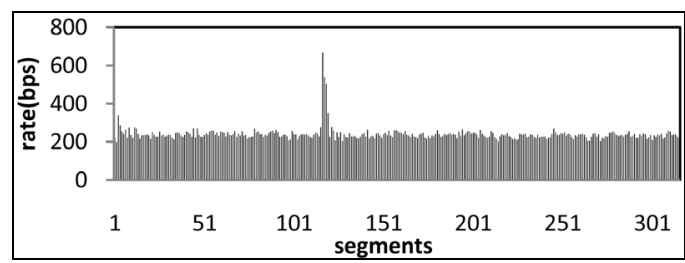

(b)

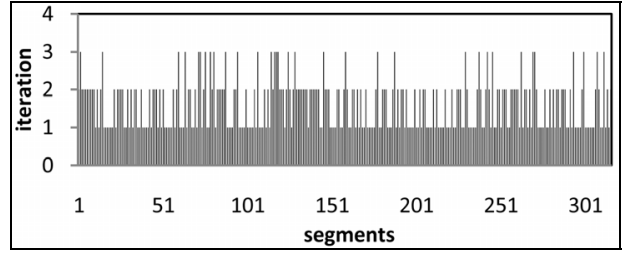

(d)

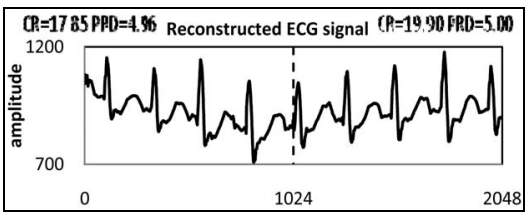

(f)

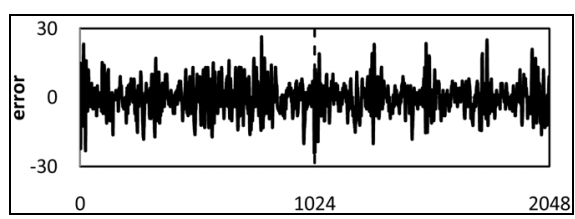

(g)

MIT-BIH Record 109

Figure 10. Performance demonstration of the proposed quantization scheme using the ECG signal of record 109 with $P R D_{T}=5 \%$ and $\varepsilon=5 \%$.

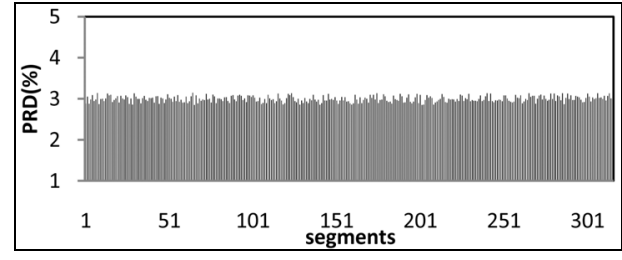

(a)

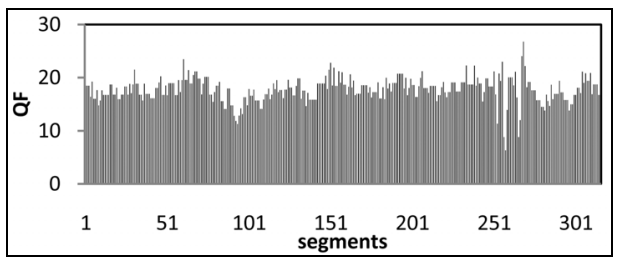

(c)

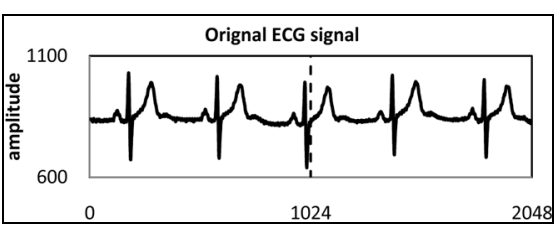

(e)

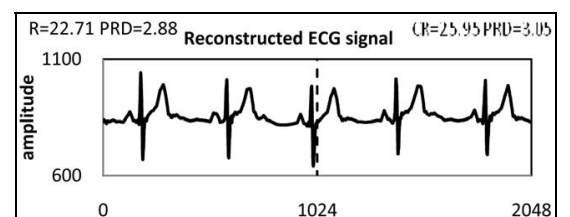

(f)

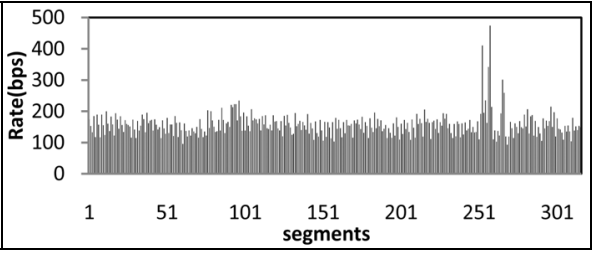

(b)

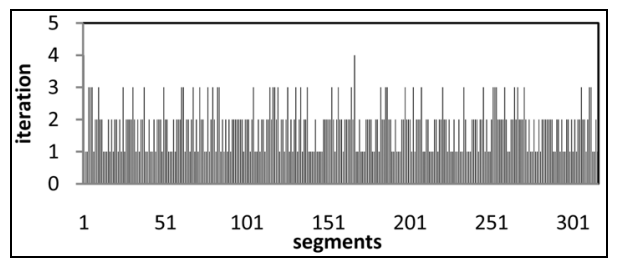

(d)

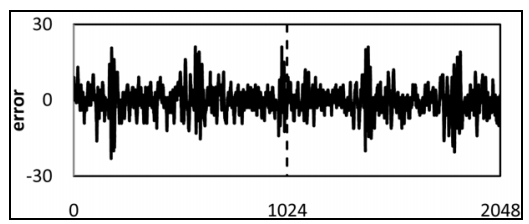

(g)

MIT-BIH Record 117

Figure 11. Performance demonstration of the proposed quantization scheme using the ECG signal of record 117 with $P R D_{T}=3 \%$ and $\varepsilon=5 \%$.

diversified signals can obtained much better compression performance, however, this improvement can be so minor. On the other hand, the quantity of training signals may be a more significant factor in overcoming signal dependency. Based on the study, a new quantization scheme with linear distortion characteristic was proposed 


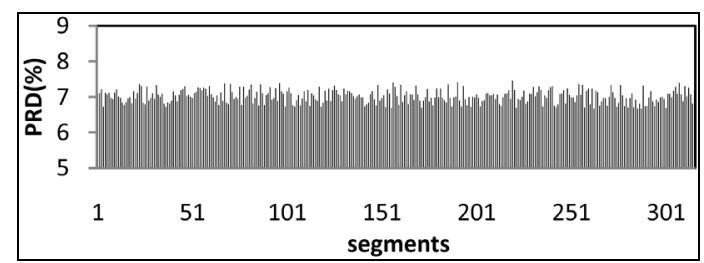

(a)

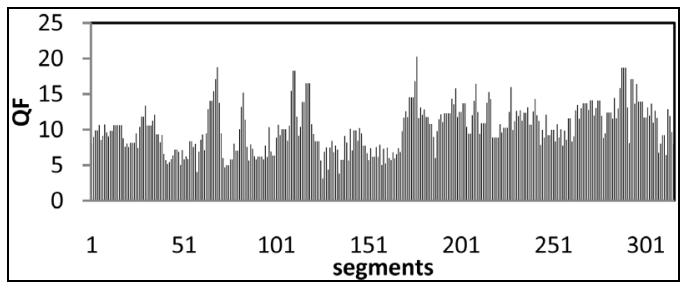

(c)

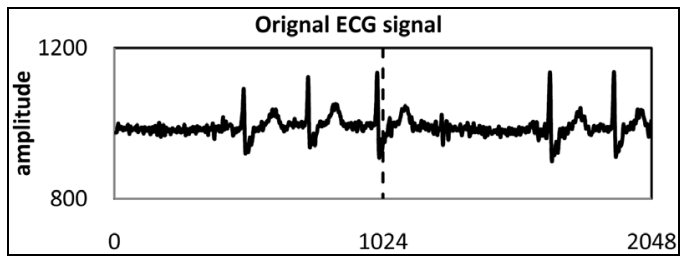

(e)

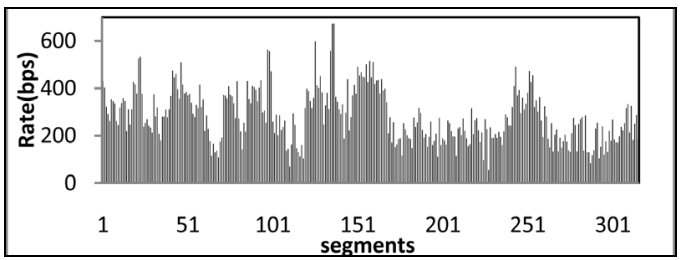

(b)

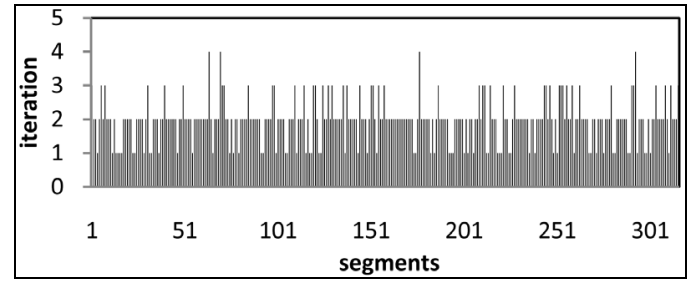

(d)

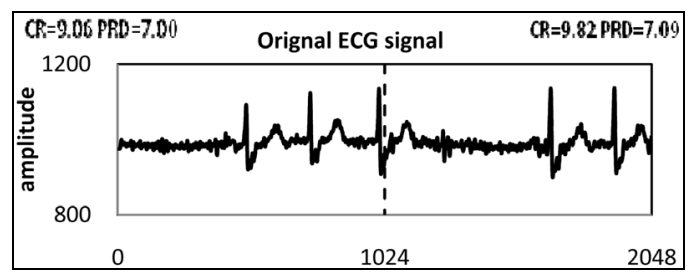

(f)

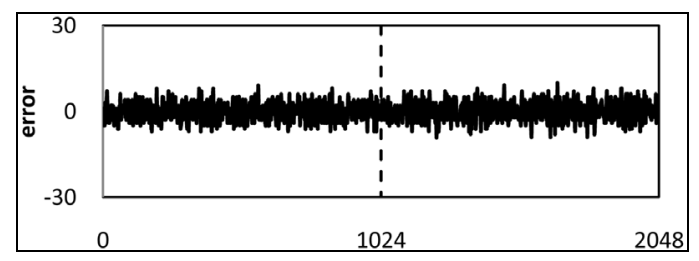

(g)

MIT-BIH Record 232

Figure 12. Performance demonstration of the proposed quantization scheme using the ECG signal of record 232 with $P R D_{T}=7 \%$ and $\varepsilon=5 \%$.

for wavelet-based ECG data compression. This quantization scheme can be easily controlled with signal variable and facilitates the issue of reconstruction quality maintenance.

\section{ACKNOWLEDGEMENTS}

The authors would like to thank the editors and refereesfor their useful comments that helped to improve this paper. This work was supported by the National Science Council ofthe Chinese Taipeiunder NSC1002221-E-327-044 and NSC101-2221-E-327-040.

\section{REFERENCES}

[1] Thaler, M.S. (2012) The ONLY EKG book you'll ever need. Lippincott Williams \& Wilkins.

[2] Ku, C.T., Hung, K.C., Wu, T.C. and Wang, H.S. (2010) Wavelet-based ECG data compression system with linear quality control scheme. IEEE Transactions on Biomedical Engineering, 57, 1399-1409.

\section{doi:10.1109/TBME.2009.2037605}

[3] Brechet, L., Lucas, M.F., Doncarli, C. and Farina, D. (2007) Compression of biomedical signals with mother wavelet optimization and best-basis wavelet packet selection. IEEE Transactions on Biomedical Engineering, 54, 2186-2192. doi:10.1109/TBME.2007.896596

[4] Al-Fahoum, A.S. (2006) Quality assessment of ECG compression techniques using a wavelet-based diagnostic measure. IEEE Transactions on Information Technology in Biomedicine, 10, 182-191. doi:10.1109/TITB.2005.855554

[5] Miaou, S.G. and Lin, C.L. (2002) A quality-on-demand algorithm for wavelet-based compression of electrocardiogram signals. IEEE Transactions on Biomedical Engineering, 49, 233-239. doi:10.1109/10.983457

[6] Zigel, Y., Cohen, A. and Katz, A. (2000) The weighted diagnostic distortion (WDD) measure for ECG signal compression. IEEE Transactions on Biomedical Engineering, 47, 1422-1430. doi:10.1109/TBME.2000.880093

[7] Lee, S.J., Kim, J. and Lee, M. (2011) A real-time ECG 
data compression and transmission algorithm for an ehealth device. IEEE Transactions on Biomedical Engineering, 58, 2448-2455. doi:10.1109/TBME.2011.2156794

[8] Lu, Z., Kim, D.Y. and Pearlman, W.A. (2000) Wavelet compression of ECG signals by the set partitioning in hierarchical trees (SPIHT) algorithm. IEEE Transactions on Biomedical Engineering, 47, 849-856. doi:10.1109/10.846678

[9] Nielsen, M., Kamavuako, E.N., Andersen, M.M., Lucas M.F. and Farina, D. (2006) Optimal wavelets for biomedical signal compression. Medical and Biological Engineering and Computing, 44, 561-568. doi:10.1007/s11517-006-0062-0

[10] He, Z. and Mitra, S.K. (2000) Optimalquantization error feedback filter for wavelet image compression. International Conference on Image Processing, 3, 166-169.

[11] Demir, B., Erturk, S. and Urhan, O. (2009) Improved quality multiple description 3D mesh coding with optimal filtering. International Conference on Image Processing, Cairo, 7-10 November 2009, 3541-3544.

[12] Batista, L.V., Melcher, E.U.K. and Carvalho, L.C. (2001) Compression of ECG signals by optimized quantization of discrete cosine transform coefficients. Medical Engineering \& Physics, 23, 127-134. doi:10.1016/S1350-4533(01)00030-3

[13] Velasco, M.B., Roldan, F.C., Llorente, J.I.G. and Barner, K.E. (2004) ECG compression with retrieved quality guaranteed. Electronics Letters, 40, 1466-1467. doi:10.1049/el:20046382

[14] Lu, Z., Kim, D.Y. and Pearlman, W.A. (2000) Wavelet compression of ECG signals by the set partitioning in hierarchical trees (SPIHT) algorithm. IEEE Transactions on Biomedical Engineering, 47, 49-856

[15] Ku, C.-T. and Hung, K.-C. (2006) A novel ECG data compression method based on nonrecursive discrete periodized wavelet transform. IEEE Transactions on Biomedical Engineering, 53, 2577-2583. doi:10.1109/TBME.2006.881772

[16] Moody, G.B. and Mark, R.G. (2001) The impact of the MIT/BIH arrhythmia database. IEEE Engineering in Medicine and Biology Magazine, 20, 45-50. doi:10.1109/51.932724

[17] Holland, J.H. (1998) Adaptation in natural and artificial systems: An introductory analysis with applications to biology, control and artificial intelligence. MIT Press, Cambridge.

[18] Golciberg, D.E. (1989) Genetic algorithms in search, optimization and machine learning. Addison-Wesley, Boston.

[19] Michalewicz, Z. (1992) Genetic algorithms + data structures $=$ evolution programs. Springer-Verlag, Berlin. doi:10.1007/978-3-662-02830-8

[20] Ku, C.-T. and Hung, K.-C. (2010) Wavelet-based ECG data compression system with linear quality control scheme. IEEE Transactions on Biomedical Engineering, 57, 1399-1409. doi:10.1109/TBME.2009.2037605 